\title{
Calidad sensorial y composición nutricional del músculo Longissimus dorsi de bovinos Cebú y sus cruces con criollo en Colombia
}

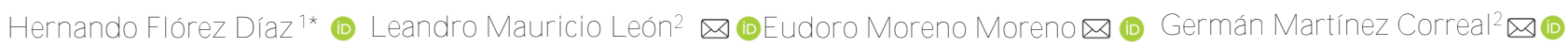 \\ Red de Ganadería y Especies Menores, Centro de Investigación La Libertad, Corporación Colombiana de Investigación Agropecuaria. \\ AGROSA VIA, Villavicencio, Meta, Colombia
}

\section{Sensory quality and nutritional composition of the Longissimus dorsi muscle of Zebu cattle and their crossbreed with creole in Colombia}

\begin{abstract}
A bstract. Shear force of the loin muscle of crossbred cattle is lower compared to Zebu cattle; however, differences in sensory quality and nutritional composition are not fully known. The objective of this study was to evaluate the sensory color, palatabilty and nutritional composition of the loin muscle of Zebu (Z), and their crossbreed with creole under conditions of the Colombian Orinoquia. Longissimus dorsi muscle steaks of $Z$ ( $n$ : 31), Sanmartinero $x$ Zebu (SM $\times C$; n: 11) and Blanco Orejinegro $\times$ Zebu $(B O N \times C ; n: 17)$ steers were used. There was found an interaction between racial group and display days with lower score on days 3 and 5 in BON $\times C$ for worst point color $(p<$ $0.0001)$, total color $(p<0.0001)$ and discoloration $(p<0.0001)$. Beef steaks of BON $\times C$ steers had lower fiber tenderness ( $p=0.0397)$ and juiciness $(p<0.0001)$ compared to other animal groups; but steaks of $Z$ steers had lower concentration of total cholesterol $(p=0.0015)$, potassium $(p=0.0257)$ and zinc $(p=0.0079)$ compared to crossbred cattle and phosphorus was lower $(p=0.0335)$ in $Z$ compared to $S M \times C$. In conclusion, differences in sensory quality and nutritional composition were found between steaks of loin muscle of the racial groups evaluated that are promising for implementing marketing and commercialization strategies that increase consumption of creole beef in Colombia.
\end{abstract}

Key words: beef, color, flavor; minerals, trained panel

Resumen. La fuerza de corte del músculo del lomo de bovinos cruzados con criollo es menor que la de bovinos Cebú (C); sin embargo, no se conocen en su totalidad las diferencias en la calidad sensorial y su composición nutricional. Este estudio tuvo como objetivo evaluar el color sensorial, la palatabilidad y la composición nutricional del músculo del lomo de bovinos $\mathrm{C}$ y sus cruces con criollo en las condiciones de la Orinoquia colombiana. Se utilizaron filetes de carne del músculo Longissimus dorsi de novillos C (n: 31), SanmartineroxCebú (SM ×C; n: 11) y Blanco OrejinegroxCebú (BON ×C; n:17). Se encontró interacción entre el grupo racial y los días de exhibición al minorista en el mostrador con menor calificación los días 3 y 5 en el BON $\times C$, para el peor punto de color $(p<$ $0.0001)$, color total $(p<0.0001)$ y decoloración $(p<0.0001)$. La carne de BON $\times C$ presentó menor calificación de la terneza de la fibra ( $p=0.0397$ ) y jugosidad ( $p<0.0001)$, pero la carne de $C$ tuvo menor concentración de colesterol total $(p=0.0015)$, potasio $(p=0.0257)$ y zinc $(p=0.0079)$ en comparación con los bovinos cruzados y el fósforo fue menor ( $p=0.0335$ ) en el $C$, con respecto al SM XC. En conclusión, se encontraron diferencias en calidad sensorial y composición nutricional de la carne de los grupos raciales evaluados que son promisorias para el desarrollo de estrategias de mercadeo y comercialización que aumenten el consumo de la carne criolla en Colombia.

Palabras Clave: carne, color, sabor, minerales, panel entrenado

Resumo. A força de cisalhamento do músculo do lombo de bovinos cruzados com criolo é menor do que a de bovnos cebus (C); no entanto, as diferenças na qual idade sensorial e na composição nutricional não são totalmente compreendidas. O objetivo deste estudo foi avaliar a cor sensorial, palatabilidade e a composição nutricional do músculo do lomo de bovinos $C$ e seus cruzamentos com o crioulo nas condições da Orinoquia colombiana. Foram utilizados filés de carne do músculo Longissimus dorsi dos novilhos C (n: 31), Sanmartinero×Cebu (SM ×C; n: 11) e Blanco OrejinegroxCebu (BON ×C; n: 17). Verificou-se interação entre o grupo racial e os dias de exposição ao lojista no balcão com a menor avaliação nos días 3 e 5 no BON $\times C$, para o pior ponto de cor ( $<\varangle 0.0001$ ), cor total. ( $p$ $\varangle 0.0001$ ) e descoloração ( $p<0.0001$ ). A carne do BON $\times C$ apresentou menor qualificação da maciez da fibra $(p=$ 0.0397 ) e suculência ( $p<0.0001)$, mas a carne do $C$ teve menor concentração de colesterol total $(p=0.0015)$, potássio $(p=0.0257)$ e zinco $(p=0.0079)$ em comparação com gado cruzado e o fósforo foi menor $(p=0.0335)$ em $C$, em

Recibido: 2020-07-30. A ceptado: 2021-02-27

*Autor para la correspondencia: hflorez@agrosavia.co

${ }^{2}$ A socriollanos, Villavicencio, Meta, Colombia. 
relação ao SM ×C. Em conclusão, foram encontradas diferenças na qualidade sensorial e na composição nutricional da carne dos grupos raciais avaliados que são promissores para o desenvolvimento de estratégias de marketing e comercialização que aumentam o consumo de carne crioula em Col ombia.

Palavras.Chave: carne, cor, gosto, minerais, painel treinado

\section{Introducción}

La producción de carne en la Orinoquia colombiana se basa principalmente en el uso de bovinos Cebú comercial (Bos indicus) en pastoreo de Brachiaria spp. A pesar de las múltiples ventajas del Cebú en términos de adaptación, hay aspectos relacionados con su temperamento, comportamiento reproductivo y calidad de la carne que son inferiores a los de razas criollas y europeas (King et al., 2006; Cafe et al., 2011; Flórez, 2011; Francisco et al., 2015; León -Llanos y Flórez-Díaz, 2016). La carne de bovinos Bos indicus presenta mayor dureza que la de bovinos Bos taurus (Crouse et al., 1989) debido a diferencias en el complejo enzimático calpaína/ cal pastatina durante la maduración (Koohmaraie y Geesink, 2006), ya que en bovinos Bos indicus es más intensa la actividad de la calpastatina que inhiben la acción de la calpaína encargada de la lisis de las fibras musculares (Hocquetteet al., 2005).

Los cruzamientos entre los bovinos criollos y europeos con Cebú se convierten en una alternativa para mejorar la eficiencia de producción durante el levante y la ceba y mejorar la calidad de la carne. Investigaciones previas demostraron que, en comparación con el Cebú comercial, el uso de los cruzamientos de novillos Bos taurus $\times$ Bos indicus durante el levante y la ceba en las condiciones del Piedemonte del Meta (Flórez, 2011; Flórez et al., 2014) aumentaron la ganancia diaria de peso corporal, disminuyeron la edad al sacrificio y las pérdidas de peso de la canal por enfriamiento; además, los cortes de carne de las canales de los bovinos cruzados presentaron menor tiempo y pérdidas por cocción (Flórez et al., 2015a).

\section{$M$ ateriales y $M$ étodos}

Los métodos y procedimientos de esta investigación fueron aprobados por el Comité Científico de la Red de Ganadería y Especies Menores de AGROSA VIA teniendo en cuenta los protocolos de cuidado y uso de animales para investigación (FUA Formato para uso de animales GA-F-191).

El trabajo se llevó a cabo en el Centro de Investigación la Libertad de AGROSAVIA en Villavicencio, Colombia, con una temperatura media de $26{ }^{\circ} \mathrm{C}$, humedad relativa del $80 \%$ y precipitación anual de $2900 \mathrm{~mm}$. Los animales del experimento fueron novillos
En una investigación posterior con base en los resultados de los concursos de ganado gordo de las ferias de San Martín y Villavicencio (Meta), se encontró que a pesar de que las canales de novillos Sanmartinero, BON $\times$ C y $\mathrm{SM} \times \mathrm{C}$ presentaron menor rendimiento en canal, estas presentaron mayor proporción de cortes finos (lomos) y total de carne aprovechable y menor proporción de hueso que las canales de novillos Cebú y Angus × Cebú (Flórez et al., 2014; Flórez et al., 2015b). Se encontró mayor relación entre el peso de la canal y la edad al sacrificio en las canales de novillos cruzados de criollos y europeos con Cebú comparadas con las de Sanmartinero y Cebú comercial. (Flórez et al., 2014). También se observó que los cortes del lomo de canales de novillos cruzados de criollo y europeo con Cebú presentaron mayor terneza, humedad y $\mathrm{pH}$, pero similar color instrumental que los de Cebú comercial (Flórez et al., 2015a; Flórez et al., 2015b).

Varias investigaciones mencionan las diferencias en la calidad sensorial y composicional de la carne de bovinos Bos indicus, Bos taurus y sus cruces finalizados en pastoreo y en lotes de engorde (Hardcastle, et al., 2017; Leheska et al., 2008, Neethling et al., 2017) y de bovinos criollos suplementados en pastoreo (Uzcátegui-Bracho et al., 2008), pero estas diferencias no se conocen en detalle en las condiciones de Colombia. El objetivo del presente trabajo fue determinar la calidad sensorial y composicional de cortes de carne de bovinos criollos, Cebú y sus cruces en las condiciones de la Orinoquia colombiana.

Cebú comercial (C; n:31), Sanmartinero $\times$ Cebú $(\mathrm{SM} \times \mathrm{C} ; \mathrm{n}: 11)$ y Blanco Orejinegro $\times$ Cebú $(B O N \times C ; n$ : 17) que finalizaron su ceba en pastoreo rotacional de praderas mixtas de Brachiaria decumbens y Brachiaria humidicola cv. dictyoneura con una carga de 1.6 animales por hectárea. Los novillos fueron suplementados en la época seca con $5 \mathrm{~kg}$ diarios por animal de ensilaje de sorgo dulce del $28 \%$ de materia seca y con un kilogramo diario por animal dos meses antes del sacrificio de un núcleo proteico energético del $14 \%$ de proteína cruda y $82 \%$ de DISMS. 
Al finalizar la ceba los novillos se sacrificaron en dos faenas, con número similar de bovinos de cada raza, en el frigorífico Guadalupe de Bogotá D.C. a $120 \mathrm{Km}$ del sitio de trabajo, con un peso vivo en pie de 463.6 $\mathrm{kg}$ y 31.3 meses para el C, $474.6 \mathrm{~kg}$ y 31.7 meses para el BON $\times$ C, y $453.4 \mathrm{~kg}$ y 32.5 meses en el $\mathrm{SM} \times \mathrm{C}$. Después del arribo a la planta de sacrificio los bovinos tuvieron un periodo de ayuno entre 18 y 24 horas con acceso libre a agua, posterior a este tiempo los animales se sacrificaron de acuerdo con los procedimientos de sacrificio humanitario requeridos por la autoridad sanitaria (INVIMA, Decretos MADR 15002007 y 2270 de 2012) de Colombia. El día del sacrificio, los bovinos se sujetaron en un brete de restricción y se insensibilizaron con una pistola neumática de perno cautivo e inmediatamente se desangraron a través de la vena yugular y arteria carótida.

A las $48 \mathrm{~h}$ post mortem durante el desposte de las canales (INVIMA Norma técnica colombiana 4291) con un peso promedio de $254.9 \pm 18.5 \mathrm{~kg}$ y rendimiento en canal fría de $55.8 \pm 2.2 \%$, se tomó de la mitad izquierda de cada canal, una sección de músculo Longissimus dorsi entre la 12 a 13 costilla con un peso aproximado de $500 \mathrm{~g}$ para la evaluación de la calidad sensorial (color y sabor) y de la composición de la carne. La sección completa del músculo se mantuvo refrigerada $\left(4\right.$ a $6{ }^{\circ} \mathrm{C}$ ) en cavas de transporte de carne que se llevaron el mismo día al laboratorio. De cada sección de músculo se tomaron filetes de $2.54 \mathrm{~cm}$ de grosor para la evaluación de color sensorial en platos de poliestireno envueltos en plástico para exhibición en vitrinas de enfriamiento (Danfoss, San Sebastián España) mantenidas por siete días entre 2 y 4 grados centígrados, sin rotación, y bajo condiciones simuladas de iluminación de supermercado (tubo LED 1540 Lm). La carne para evaluación de palatabilidad se preparó en filetes de $2.54 \mathrm{~cm}$, que se empacaron al vacío en bolsas plásticas con una empacadora el vacío de mesa (Multivac Baseline P 200, Nettetal. Germany) y se maduraron en nevera por 14 días entre 2 y $4{ }^{\circ} \mathrm{C}$. Después de la maduración los cortes de carne se congelaron a $-20{ }^{\circ} \mathrm{C}$ hasta su procesamiento. Los filetes de carne $(2.54 \mathrm{~cm})$ para la determinación de la composición nutricional, el día del desposte, se almacenaron al vacío y se congelaron a $-20^{\circ} \mathrm{C}$ hasta su análisis.

\section{Evaluación sensorial de color}

Para la evaluación se utilizaron ocho panelistas previamente entrenados de acuerdo con las normas de la Asociación Americana de la Ciencia de la Carne (AMSA, 2012). Durante los días 1, 3, 5, y 7 de exposición simulada en vitrinas de enfriamiento, de acuerdo con los métodos descritos por Baublits et al. (2006a), los panelistas evaluaron el peor punto de color (en un área del filete de aproximadamente $1 \mathrm{~cm}$ de diámetro) y el color total (en el total de la superficie del filete) en una escala de 1 a 5 ( $5=$ rojo púrpura brillante, 4= rojo púrpura pálido, $3=$ levemente rojizo café, $2=$ moderadamente rojizo café, y $1=$ café); además, evaluaron el porcentaje de decoloración superficial en la totalidad del filete $(7=\sin$ decoloración (0\%), 6= decoloración leve (1 - $20 \%), 5=$ decoloración pequeña (20 - $39 \%$ ), 4= decoloración modesta (40 - $59 \%$ ), 3= decoloración moderada (60 $79 \%), \quad 2=$ decoloración extensa (80 - $95 \%$ ), 1= decoloración total (96 - $100 \%$ ). Cada panelista registro su evaluación en formatos que contenían Cuadros para cada parámetro evaluado y las guías de calificación. Los registros de la evaluación de cada panelista se almacenaron en bases de datos utilizando el programa Excel de Windows.

\section{Evaluación sensorial de la Palatabilidad de la Carne}

Los filetes previamente preparados $(2.54 \mathrm{~cm}$. de grosor) se descongelaron por $24 \mathrm{~h}$ a $4{ }^{\circ} \mathrm{C}$, se retiraron de sus bolsas al vacío y se cocinaron en un horno de convección de aire forzado (Unox modelo XFO43 Serie 2528, Bogotá Colombia) hasta que la temperatura interna de cada filete alcanzó $70^{\circ} \mathrm{C}$. La temperatura se monitoré usando termo sondas (Hanna Instruments HI98509, Bogotá, Colombia). Inmediatamente después de la cocción, los filetes se cortaron en cubos de $1 \times 1 \times$ $1 \mathrm{~cm}$. y se mantuvieron en el horno a $60{ }^{\circ} \mathrm{C}$ por 15 minutos antes de la evaluación sensorial y durante el proceso de evaluación. Para la evaluación, los cubos de carne se sirvieron tibios a un panel de ocho personas entrenadas según los métodos descritos por la AMSA, (2016) y los descritos por Baublits et al. (2006b).

Los panelistas para la evaluación de sabor se seleccionaron verbalmente y con base en su gusto por la carne bovina y su deseo de participar en dos sesiones diarias (mañana y tarde con diferencias de 5 horas entre sesiones) por tres días por tres semanas no continuas. Las personas del panel se entrenaron en tres sesiones de dos horas cada una antes de la evaluación con varias muestras de carne y se escogieron con base en su habilidad para determinar diferencias en la terneza, la jugosidad y la intensidad del sabor de la carne. Al menos la mitad de los panelistas tenían una experiencia previa de 80 horas.

Cada día, una réplica de las muestras de músculo se sirvió al eatoriamente a cada panelista de tal forma que todos los panelistas evaluaron muestras de todas las 
razas en cada sesión. Las muestras se sirvieron al azar a cada panelista que las evaluaron sin restricciones de tiempo. Los panelistas (8) evaluaron la terneza de la fibra y la terneza general, la cantidad de tejido conectivo, jugosidad y la intensidad de sabor en una escala hedónica de 8 puntos. ( 1 = extremadamente duro, abundante, extremadamente seco, extremadamente sin intensidad de sabor a carne; 8 = extremadamente tierno, ninguno, extremadamente jugoso, extremadamente intenso sabor a carne). A dicionalmente, los panelistas analizaron las muestras para detectar la intensidad de sabores desagradables según lo descrito por Baublits et al. (2006b), en una escala hedónica de 5 puntos ( 1 = sabor extremo, 2 = sabor moderado, 3 = sabor pequeño, 4 = sabor leve, 5 = no sabor desagradable). Entre cada muestra evaluada los panelistas consumieron una galleta de soda sin sal para remover el sabor residual y tomaron agua a temperatura ambiente para limpiar la boca. Las evaluaciones se realizaron bajo condiciones de luz natural en puestos separados para aislar a cada evaluador. Cada panelista registro su evaluación en formatos que contenían Cuadros para cada parámetro evaluado y las guías de calificación. Los registros de la evaluación de cada panelista se almacenaron en bases de datos utilizando el programa Excel de Windows.

\section{Composición Nutricional de la Carne}

Los cortes de carne se descongelaron por $24 \mathrm{~h}$ a $4{ }^{\circ} \mathrm{C}$ y se evaluaron para materia seca, humedad, proteína cruda, grasa (extracto etéreo) y cenizas de acuerdo con los métodos descritos por la AOAC (AOAC, 1990). La concentración de colesterol se determinó por cromatografía líquida de alta eficiencia HPLC., siguiendo el método descrito por Mestre Prates, et al. (2006). La estabilidad oxidativa del músculo Longissimus dorsi después del almacenamiento a $4{ }^{\circ} \mathrm{C}$ por $24 \mathrm{~h}$, se determinó por el método de determinación de las sustancias reactivas del ácido tiobarbitúrico (TBARS) por espectrofotometría siguiendo el protocolo descrito por Monin et al . (2003) citados por Rossi et al. (2013). La concentración de TBARS se expresó como los miligramos de malondialdehído (MDA) por kilogramo de carne. La concentración de calcio, sodio, magnesio, potasio, hierro y zinc se determinó mediante espectrofotometría siguiendo los métodos descritos por la AOAC (AOAC, 1997).

\section{Análisis Estadístico}

La información se analizó utilizando el paquete de análisis estadístico SAS (SAS Inst. Inc., Cary, NC) utilizando el procedimiento MIXED de SAS. El color sensorial se analizó utilizando análisis de medidas repetidas a lo largo de los días de exposición de cada tipo de animal. El panel sensorial de sabor se analizó en un modelo completamente al azar con el tipo de animal con efecto fijo y el panelista como efecto aleatorio. Un modelo similar se utilizó para la información de composición nutricional con el tipo de animal como efecto fijo. Las medias de tratamiento y sus interacciones se compararon utilizando la opción PDIFF de SAS. Los cuadrados medios mínimos se consideraron estadísticamente significativos al nivel de $P \leq 0.05$.

\section{Resultados y Discusión}

\section{Evaluación Sensorial de Color}

En la Figura 1 se presentan los cambios en color evaluados por los panelistas entrenados. La calificación del peor punto del color (área de cambio de color de al menos $1 \mathrm{~cm}$ de diámetro) en la superficie del filete por tipo de animal (Figura 1) presentó una interacción $(p<0.0001)$ entre el grupo racial y los días almacenamiento (exposición a las condiciones simuladas de supermercado). Los filetes del BON $\times$ C tuvieron menor puntuación los días 3 y 5 con relación al Cebú y SM $\times$, pero en los días 1 y 7 no hubo diferencias en la puntuación del peor punto de color entre grupos raciales.

En forma similar, la calificación del color total (área total de cambio de color) en la superficie del filete (Figura 2) encontró interacción ( $p<0.0001$ ) entre el grupo racial y los días almacenamiento. Los filetes de BON $\times$ C tuvieron menor puntuación de color total en los días 3 y 5 con relación al Cebú y $\mathrm{SM} \times \mathrm{C}$, pero los días 1 y 7 no hubo diferencias en la puntuación del color total entre grupos raciales.

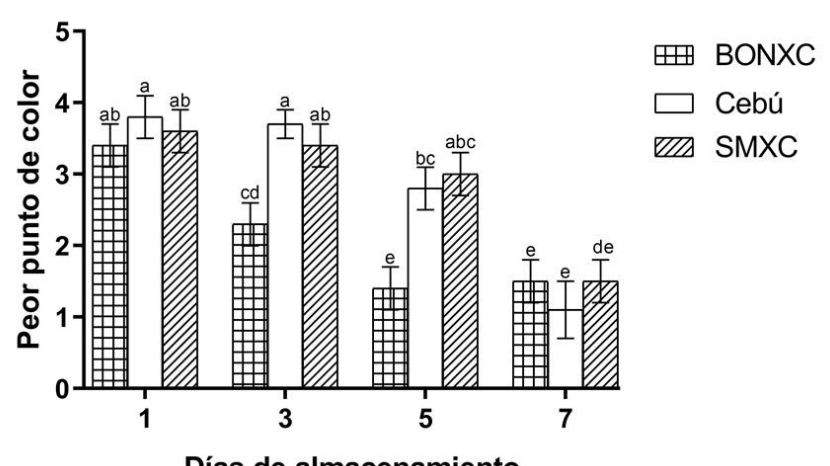

'Evaluación de 1 a 5 (5= rojo púrpura brillante, 4= rojo púrpura pálida, 3= levemente rojizo café, $2=$ moderadamente rojizo café, y $1=$ café)

Figura 1. Interacción entre el grupo racial y los días de almacenamiento para el peor punto de color ${ }^{1}$ de cortes del músculo Longissimus dorsi de diferentes grupos raciales evaluados por panelistas entrenados del durante el día 1 a 7 de exposición aerobia a $4{ }^{\circ} \mathrm{C}$ en vitrinas de enfriamiento. 


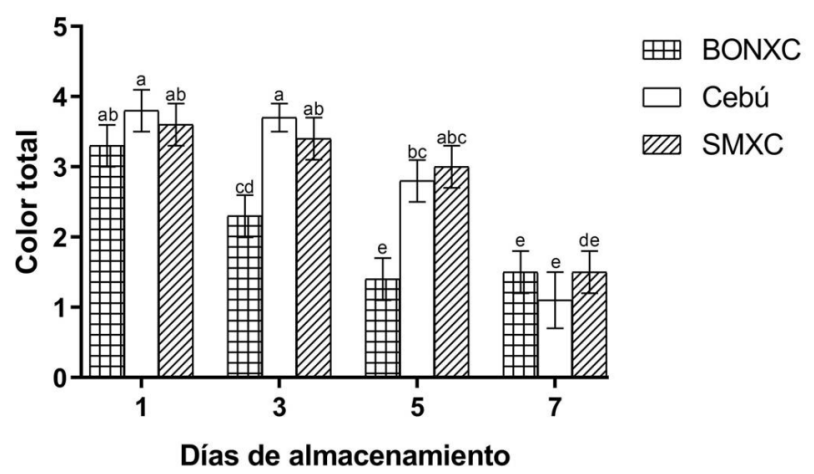

${ }^{1}$ Evaluación de 1 a 5 (5= rojo púrpura brillante, 4= rojo púrpura pálido, 3= levemente rojizo café, $2=$ moderadamente rojizo café, y $1=$ café)

Figura 2. Interacción entre el grupo racial y los días de almacenamiento para el color total ${ }^{1}$ de cortes del músculo Longissimus dorsi de diferentes grupos raciales evaluados por panelistas entrenados del durante el día 1 a 7 de exposición aerobia a $4{ }^{\circ} \mathrm{C}$ en vitrinas de enfriamiento.

La calificación de la decoloración (área total de cambio de color) en la superficie del filete (Figura 3) presentó interacción $(p<0.0001)$ entre el grupo racial y los días almacenamiento. Los filetes de BON $\times C$ tuvieron mayor decoloración los días 3 y 5 en comparación con el SM $\times C$ y Cebú, pero no hubo diferencias entre grupos raciales los días 1 y 7 ( $p>$ 0.05).

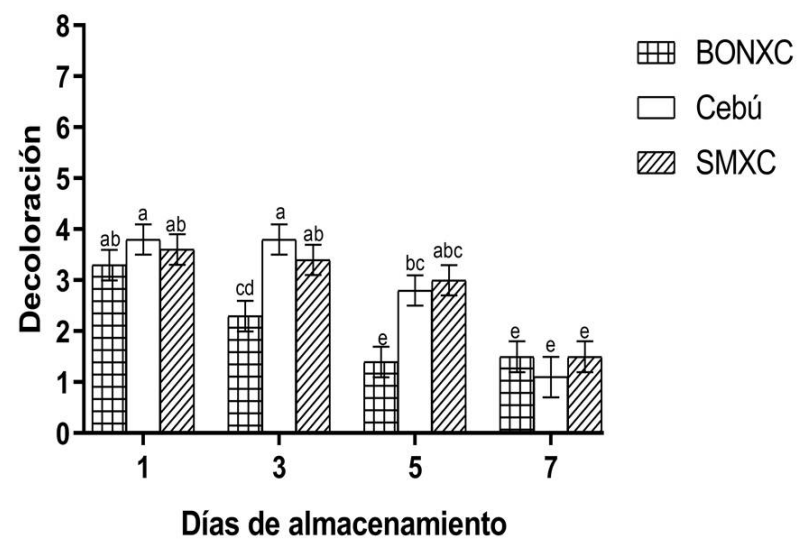

Porcentaje de decoloración superficial $(7=\sin$ decoloración $(0 \%), 6=$ decoloración leve (1-20 \%),5= decoloración pequeña (20-39 \%), 4= decoloración modesta (40-59\%), 3= decoloración moderada (60-79\%),2= decoloración extensa (80-95\%), 1= decoloración total (96-100\%).

Figura 3. Interacción entre el grupo racial y los días de almacenamiento para el porcentaje de decoloración1 de cortes del músculo Longissimus dorsi de diferentes grupos raciales evaluados por panelistas entrenados durante el día 1 a 7 de exposición aerobia a $4{ }^{\circ} \mathrm{C}$ en vitrinas de enfriamiento.

La primera impresión que los consumidores tienen de un corte de carne es su color, por lo que éste se constituye en un aspecto de gran importancia. Los consumidores relacionan el color con la frescura de la carne y de hecho este se relaciona con la decisión de compra (Mancini y Hunt, 2005; Suman et al., 2014). Además, se conoce que las personas también relacionan el color con el sabor, la inocuidad y el valor nutritivo de la carne (Girolami et al., 2013). El color que perciben las personas está determinado por múltiples factores intrínsecos y extrínsecos como la concentración y el estado de oxidación de la mioglobina, la morfología de la estructura muscular, el pH, la forma con la que el corte de carne absorbe o difracta la luz incidente sobre músculo, pero también por la raza, edad y sexo entre otros aspectos (Neethling et al., 2017; Miguel et al., 2014; Seidemani et al., 1984).

La menor puntuación en el peor punto de color (moderamente marrón), color total (ligeramente marrón) y decolaración (entre el 40 y $60 \%$ del filete) que los panelistas entrenados les dieron a los filetes de lomo de bovinos BON $\times$ C puede estar relacionada con el tipo de animal y el manejo. King et al. (2014) mencionan diferencias entre razas ya que en general, los filetes de carne de canales de bovinos con influencia de raza Charolais y Limousin tiene mayor estabilidad en el color comparadas con Angus, Hereford, frente a otras con estabilidad intermedia como el Simmental y Gelbvieh. Estas diferencias entre razas se pueden explicar por diferencias en la actividad metabólica muscular. Cuvelier et al. (2006) encontraron que la carne de toros Angus tenía mayor actividad de la enzima citocromo c oxidasa y menor actividad de lactato deshidrogenasa y en la evaluación instrumental de color menor luminosidad $\left(L^{*}\right)$ que cortes de toros Limousin y Blanco Azul Belga.

En sistemas extensivos de pastoreo Vestergaard et al. (2000) describieron aumento de la proporción de fibras musculares $\alpha$ rojas y de la concentración de pigmentos y a nivel instrumental bajos valores de luminosidad ( $\left.L^{*}\right)$ y de color rojo (a*) en cortes del músculo Longissimus comparados con bovinos en sistemas intensivos. May et al. (1977) encontraron que la carne de novillos Limousin $\times$ Angus tenían mayor proporción de fibras musculares $\alpha$ blancas en el músculo Longissimus que la de novillos Hereford $\times$ Angus y Simmental $\times$ Angus. Estos hallazgos pueden explicar parcialmente las diferencias entre grupos raciales descritas en esta investigación.

Llama la atención que los panelistas entrenados pudieron detectar diferencias en estos cortes en forma similar a la encontrada previamente cuando se evaluó la luminosidad ( $L *)$ y la intensidad de color rojo (a*), los cual fue menor en el BON $\times C$ y SM ×C y mayor en el Cebú (Flórez et al., 2015). Estos resultados también son similares a los reportados en filetes de carne de bovinos Bos in dicus de raza N el ore 
y cruces Bos indicus $\times$ Bos taurus de Nelore $\times$ Angus finalizados en lotes de engorde (Gómez et al., 2017; Miguel et al., 2014).

En forma similar a lo descrito en el presente estudio, en bovinos Bos indicus (Nelore) sometidos a diferentes procesos de sacrificio, se encontró que la intensidad de color rojo (a*) y la estabilidad de color de cortes del músculo Longissimus se pierde rápidamente a partir del día 3 de almacenamiento a $4{ }^{\circ} \mathrm{C}$ (Salim et al., 2019). Los hallazgos del presente trabajo también son similares a los reportados por Hyun-Wook et al. (2016) en cortes del músculo Longissimus lumborum de canales de bovinos Bos taurus quienes encontraron diferencias en el color total y la decoloración a partir del día 4 de exposición en condiciones simuladas de supermercado.

Uno de los aspectos de importancia observados en esta investigación, es que, bajo nuestras condiciones, a partir del día 3 de exposición a las condiciones simuladas de supermercado, la percepción y la puntación dada por los panelistas a los cortes de lomo disminuye con relación al día 1 especialmente para los cortes de BON $\times$ C comparados con Cebú y $\mathrm{SM} \times \mathrm{C}$ (Figuras 1 y 2), pero ya al día 7 de exposición las calificaciones del peor punto de color (marrón), color total (moderamente rojo marrón) y decoloración (entre el 60 y $80 \%$ ), no son diferentes entre grupos raciales (Figuras 1, 2 y 3).

Lo anterior puede indicar que los cortes de carne del músculo Longissimus dorsi de bovinos del presente estudio, entre el tercer y quinto día de exposición a condiciones simuladas de supermercado pierden valoración por parte de panelistas entrenados, lo que eventualmente puede afectar la decisión de compra. De hecho, se ha demostrado en varios estudios que los consumidores están dispuestos a pagar por cortes y filetes de carne con color rojo cereza brillante (Font-iFurnols y Guerrero, 2014; Gracia y de Magistris, 2013; Udomkun et al., 2018), ya que los colores oscuros se asocian con mala calidad y contaminación de la carne (Mancini y Hunt, 2005, N eethling et al., 2017).

En el caso del BON $\times$ C se debe tener mayor atención ya que los cortes de carne pierden las características deseables de color incluso desde el día tres de exposición. Los encargados del manejo de productos cárnicos en los supermercados y expendios de carne deben tener en cuenta las diferencias entre grupos raciales y tiempos de exposición descritas en este trabajo para ajustar los procesos de venta y comercialización de la carne.

\section{Evaluación Sensorial de la Palatabilidad de la Carne}

La evaluación sensorial de la palatabilidad de los filetes de carne según el grupo racial se presenta en el Cuadro 1. Se encontró que los filetes de carne del músculo Longissimus dorsi de bovinos $\mathrm{BON} \times \mathrm{C}$ presentaron menor calificación de la terneza de la fibra $(p=0.0397)$ y la jugosidad $(p<0.0001)$ comparadas con la carne de $\mathrm{C}$ y $\mathrm{SM} \times \mathrm{C}$. Así mismo, se encontró en los filetes de BON $\times \mathrm{C}$ menor calificación de la terneza general ( $p=0.0378$ ) en comparación con los bovinos Cebú, y tendieron ( $p=0.0749)$ a tener menor terneza general con relación a los de SM×C. También se observó que los panelistas calificaron la intensidad de sabores desagradables mayor $(p=0.0371)$ en los filetes de carne del $\mathrm{SM} \times \mathrm{C}$ que en el $\mathrm{BON} \times \mathrm{C}$, pero no con los de $C$. A unque no hubo diferencias, los filetes de carne del BON $\times C$ tendieron a presentar mayor $(p=0.0975)$ cantidad de tejido conectivo y menor $(p=0.0948)$ intensidad de sabor comparados con los de $C$, pero no con los de SM XC.

Cuadro 1. Calidad Sensorial de la palatabilidad de filetes de carne del músculo L ongissimus dorsi en Bovinos Cebú, Sanmartinero $\times$ Cebú $(\mathrm{SM} \times \mathrm{C})$ y Blanco Orejinegro ×Cebú $(\mathrm{BON} \times \mathrm{C})$ evaluada por panelistas entrenados.

\begin{tabular}{lcccr}
\hline Variable & \multicolumn{3}{c}{ Grupo Racial } \\
\cline { 2 - 5 } & Cebú & SM $\times C$ & BON $\times C$ & Pr $>$ F \\
\hline Terneza dela fibra $^{1}$ & $4.91 \pm 0.15^{a^{*}}$ & $4.92 \pm 0.18^{\mathrm{a}}$ & $4.40 \pm 0.17^{\mathrm{b}}$ & 0.0397 \\
Cantidad de Tejido Conectivo $^{1}$ & $4.82 \pm 0.14^{\mathrm{a}}$ & $4.65 \pm 0.15^{\mathrm{a}}$ & $4.49 \pm 0.14^{\mathrm{a}}$ & 0.2516 \\
Terneza General $^{1}$ & $4.96 \pm 0.14^{\mathrm{a}}$ & $4.91 \pm 0.16^{\mathrm{ab}}$ & $4.53 \pm 0.14^{\mathrm{b}}$ & 0.0818 \\
Jugosidad $^{1}$ & $4.88 \pm 0.12^{\mathrm{a}}$ & $4.69 \pm 0.13^{\mathrm{a}}$ & $4.06 \pm 0.13^{\mathrm{b}}$ & $<0.0001$ \\
Intensidad de Sabor $^{1}$ & $5.21 \pm 0.11^{\mathrm{a}}$ & $5.16 \pm 0.12^{\mathrm{a}}$ & $4.95 \pm 0.11^{\mathrm{a}}$ & 0.2165 \\
Intensidad de Sabores desagradables $^{2}$ & $4.71 \pm 0.07^{\mathrm{ab}}$ & $4.59 \pm 0.08^{\mathrm{b}}$ & $4.81 \pm 0.05^{\mathrm{a}}$ & 0.1127 \\
\hline
\end{tabular}

a,bM edias con diferente letra entre columnas, difieren entre sí ( $\mathrm{P} \leq 0.05)$.

'Escala de 1 a 8 donde 1 es dura, abundante, muy seca y sin intensidad de sabor a carne y 8 es muy blanda, ausente, muy jugosa y con alta intensidad de sabor a carne.

Escala de 1 a 5 donde 1 es con alta intensidad de sabor desagradable y 5 es sin intensidad de sabor desagradable. 
La terneza, la jugosidad y el sabor afectan la palatabilidad de la carne. Entre estas características la terneza es el factor más importante para los consumidores de carne (Miller et al., 2001) y se conoce que las personas están dispuestas a pagar más por carne que garantice su terneza (Boleman et al., 1997). La terneza se asocia con facilidad de morder y masticar la carne.

Con respecto a los resultados generales del panel sensorial del músculo Longissimus dorsi de este trabajo se encontraron filetes de carne con valores intermedios para terneza (entre algo dura y algo tierna), cantidad de tejido conectivo (entre algo escaso y algo abundante), jugosidad (entre algo seca y algo jugosa), intensidad de sabor a carne (con algo de intensidad de sabor) y la intensidad de sabores desagradables (sin sabores desagradables).

En Venezuela Huerta-Leidenz et al. (2004), reportaron valores similares del panel sensorial para filetes del músculo Longissimus madurados al vacío por 14 días de toros F1 Senepol $\times$ Cebú que se mantuvieron en praderas de Brachiaria humidicola, Brachiaria plantaginea y sabana nativa (Paspalum fasciculatum, Leersia hexandra and Hymenachne amplexicaulis) con suplementación mineral. Así mismo en Venezuela, Connell et al. (2002) reportaron en filetes del músculo Longissimus thoracis valores similares de evaluación sensorial y no encontraron diferencias entre grupos raciales de bovinos del sistema de doble propósito de la región del Zulia clasificados como mestizos Cebú, mestizos lecheros y mestizos indefinidos.

En Brasil Fruet et al. (2018), en novillos cruzados (Hereford, Angus y Nelore) de 18 a 20 meses con un peso inicial de $333 \mathrm{~kg}$, que tuvieron su levante en pastoreo y la ceba en pasturas asociadas de gramíneas y leguminosas y suplementación con granos observaron calificaciones similares de los panelistas en la evaluación sensorial de la terneza de la fibra y general (cortes entre algo duros y algo tiernos), jugosidad (algo secos, algo jugosos), intensidad de sabor (con sabor) y la intensidad de sabores desagradables (sin sabores desagradables) del músculo Longissimus thoracis.

Así mismo, Merle et al. (2004) en un trabajo realizado en Venezuela en bovinos Bos indicus Cebú que se manejaron en pastoreo de praderas de Brachiaria spp al igual que este trabajo, encontraron valores similares a los reportados para el BON $\times$ C en la terneza de la fibra, la cantidad de tejido conectivo y la terneza general. Pavan y Duckett (2019) en Estados Unidos, también reportaron valores similares a los resultados del panel sensorial de este trabajo en filetes del músculo Longissimus thoracis de bovinos Bos taurus Angus en pastoreo y en pastoreo con suplementación de granos.

Por el contrario, Rodas-González et al. (2007) en filetes del músculo Longissimus madurados por 14 días de novillos criollo limonero de 36 meses de edad, que pastoreaban praderas de Echinochloa polystachia con y sin suplementación y ramoneo de Leucaena leucocephala encontraron mayor valor de la calificación de la blandura y menor intensidad de sabor a la reportada en el presente estudio; de igual forma, Bueso et al., 2018 , en cortes de músculo del lomo madurados por 21 días de bovinos Bos indicus de tres años finalizados en pastoreo en Honduras, encontraron menores calificaciones por los consumidores a la terneza de la fibra y general (cortes algo duros), jugosidad (algo secos) e intensidad de sabor (sin sabor) que las encontradas en la presente investigación.

Con relación al efecto del grupo racial, el presente estudio mostró que los cortes de carne del músculo Longissimus dorsi de bovinos $\mathrm{BON} \times \mathrm{C}$ evaluados por panelistas entrenados fueron calificados como algo duros en la terneza de la fibra y general y como menos jugosos en comparación con los otros grupos raciales. La mayor dureza de la carne del BON $\times$ C contrasta con la menor terneza de los cortes de lomo de Cebú que se reportó en estudios previos en la región (Flórez, 2011).

Contrario a lo encontrado en esta investigación, la evaluación sensorial de filetes de carne en bovinos Bos indicus Nelore y de la raza Canchim (cruce de Cebú por Charolais) en Brasil, no se encontraron diferencias en la terneza general, intensidad de sabor y preferencia por los consumidores entre grupos raciales (Fernandes et al., 2009). En igual forma, Hardcastle, et al. (2017) reportaron que los consumidores en Honduras no encontraron diferencias en la palatabilidad de filetes de lomo de bovinos Bos indicus y sus cruces finalizados en pastoreo o suplementados durante la ceba con granos de destilería, torta de palmiste o caña de azúcar. Estos resultados variables se pueden explicar en parte con los hallazgos de Malheiros et al. (2015) en bovinos Nelore finalizados en lotes de engorde donde se observó alta variabilidad en los resultados de terneza general, lo que afectó la percepción del sabor en los consumidores y la calidad del producto final.

La percepción por parte de los panelistas de la mayor dureza de los filetes de carne en el $\mathrm{BON} \times \mathrm{C}$ que se observó en esta investigación, se puede relacionar con la menor jugosidad y posiblemente a la tendencia a calificar estos filetes con mayor cantidad de tejido 
conectivo en especial cuando se compraron con los de C (Cuadro 1). La jugosidad se puede relacionar con factores como el $\mathrm{pH}$, la tasa de desnaturalización de las proteínas durante la cocción, las diferencias en la capacidad de retención de agua y el grado de cocción (A berle et al., 1981; Lorenzen et al., 1999; Lucherk et al., 2016).

En un estudio previo en la región de la Orinoquia se encontraron diferencias en la capacidad de retención agua, pero no en el pH y las pérdidas por cocción entre grupos raciales (Flórez, 2011). A pesar de que la percepción de jugosidad también se relaciona con la concentración de la grasa intramuscular (Emerson et al., 2013; Lucherk et al., 2016, Nyquist et al., 2018), en el presente estudio no se observaron diferencias entre grupos raciales en la concentración de grasa intramuscular (Cuadro 2) que explicaran esta relación. Por otra parte, Miller et al. (2001) determinaron que se debe dar énfasis a la jugosidad y el sabor a medida que la terneza disminuye, ya que la satisfacción de los consumidores de filetes duros tienden a asociarla más a la jugosidad y el sabor, por lo que es posible que los cortes de carne más secos del BON $\times C$ afectaran la cal ificación de la terneza dada por los panel istas.

El tejido conectivo intramuscular (TCI) en la carne está compuesto principalmente por fibras de colágeno y elastina rodeadas de una matriz de proteoglicanos (Purslow, 2005; Astruc, 2014). La concentración, tipo y solubilidad del colágeno se relacionan directamente con la terneza de la carne. En estudios de metaanálisis y de revisión de las relaciones entre la terneza sensorial del músculo Longissimus thoracis y la cantidad de tejido conectivo, se observó que la concentración total de colágeno fue un factor relacionado positivamente con la dureza de la carne (Chriki et al., 2013; Astruc, 2014).

Se ha reportado que la raza afecta la concentración de colágeno del músculo debido a las diferencias en las tasas de madurez fisiológica entre razas (Roy et al., 2015; Bruce and Roy, 2019). En trabajos para determinar el efecto del porcentaje de genes Brahman (Bos indicus) en novillos cruzados con Angus (Bos taurus) mantenidos en pastoreo hasta el destete y posteriormente en una dieta completa de maíz y granos hasta el sacrificio, sobre la palatabilidad de la carne del músculo Longissimus lumborum madurado al vacío por 14 días, Phelps et al. (2017), encontraron que a medida que aumentaba la proporción de sangre Brahman, la calificación de la terneza general dada por panelistas entrenados disminuía y la calificación de la cantidad de tejido conectivo aumentaba. En este mismo estudio, la calificación de los panelistas de la terneza general, la jugosidad y el tejido conectivo fueron correlacionadas negativamente con el porcentaje de genética Brahman.

Es posible que, en el BON por ser una raza que se formó en la zona montañosa de la región cafetera de Colombia (Martínez y Gómez, 2006), se puedan encontrar animales puros y cruzados que produzcan carne con mayor tejido conectivo y dureza, asociado al mayor esfuerzo muscular de los bovinos que pastorean en áreas de ladera. De hecho, se conoce que uno de los factores que pueden afectar la cantidad y composición del $\mathrm{TCl}$ es el ejercicio el cual aumenta la cantidad de este tejido (Purslow, 2005).

Sin embargo, otros autores como Astruc (2014) en su revisión sobre la estructura y función del tejido con relación a la calidad de la carne bovina, se menciona que no se encuentran diferencias consistentes en el colágeno y su solubilidad entre razas bovinas; a pesar de que las razas Bos indicus tienen mayor concentración de colágeno y menor solubilidad, lo cual sugiere según el autor que el colágeno afecta la terneza de la carne solo entre líneas puras de Bos indicus. En igual forma, Martins et al. (2015) no encontraron diferencias en la concentración de colágeno de bovinos Angus y N elore en Brasil.

\section{Composición Nutricional de la Carne}

La concentración de materia seca, humedad, proteína total, extracto etéreo, cenizas, colesterol total y las sustancias reactivas del ácido tiobarbitúrico (TBARS) del músculo Longissimius dorsi de los diferentes grupos raciales evaluados se presenta en el Cuadro 2. El colesterol total fue menor ( $p=0.0015$ ) en los cortes del músculo Longissimus dorsi de bovinos Cebú en comparación con los cortes de BON XC y SM XC. Para demás variables evaluadas no se encontraron diferencias $(p>0.05)$ entre los grupos raciales evaluados (Cuadro 2).

La carne bovina está compuesta por proteínas de al to valor biológico y micronutrientes esenciales como minerales y vitaminas de importancia para la buena salud de los consumidores. La carne también contiene grasas incluyendo los ácidos grasos mono y poliinsaturados. Actualmente, los consumidores se interesan en conocer la composición nutricional de la carne especialmente en la cantidad de grasa (Scollan et al., 2006) debido a los estudios relacionados con el efecto negativo en la salud (Lippi et al., 2015). Algunos de los factores que influyen en la composición nutricional de la carne bovina son la raza, el sistema de alimentación, la época del año y el tipo de corte 
evaluado (Williams, 2007; Freitas et al., 2014); sin embargo, para el caso de la proténa no se han encontrado efectos de la nutrición y la composición racial en la concentración de proteína y el perfil de aminoácidos de la carne bovina (Scollan et al., 2006; Leheska et al., 2008; Van Elswyk et al., 2014).

Cuadro 2. Concentración de Materia Seca, Humedad, Proteína Total, Extracto Etéreo, Cenizas, Colesterol Total y Sustancias Reactivas del Ácido Tiobarbitúrico (TBARS) en filetes del músculo Longissimus dorsi de bovinos Cebú, Sanmartinero $\times$ Cebú $(\mathrm{SM} \times \mathrm{C})$ y Blanco Orejinegro $\times$ Cebú $(\mathrm{BON} \times \mathrm{C})$

\begin{tabular}{|c|c|c|c|c|}
\hline \multirow[t]{2}{*}{ Variable } & \multicolumn{4}{|c|}{ Grupo Racial } \\
\hline & Cebú & $\mathrm{BON} \times \mathrm{C}$ & $\mathrm{SM} \times \mathrm{C}$ & $\mathrm{Pr}>\mathrm{F}$ \\
\hline Materia Seca \% & $27.98 \pm 0.75^{a}$ & $28.33 \pm 0.32^{a}$ & $28.81 \pm 0.46^{a}$ & 0.5676 \\
\hline Humedad, \% & $72.02 \pm 0.75^{a}$ & $71.67 \pm 0.52^{a}$ & $71.19 \pm 0.46^{a}$ & 0.5676 \\
\hline Proteína Cruda, \% & $17.10 \pm 1.60^{\mathrm{a}}$ & $17.68 \pm 0.82^{a}$ & $19.51+1.66^{a}$ & 0.5199 \\
\hline Extracto Etéreo, \% & $6.91+1.58^{a}$ & $6.27 \pm 1.52^{a}$ & $4.43 \pm 1.54^{a}$ & 0.6252 \\
\hline Cenizas, \% & $0.89 \pm 0.08^{a}$ & $0.84 \pm 0.04^{a}$ & $0.89 \pm 0.07^{a}$ & 0.7953 \\
\hline Colesterol Total, mg/ 100g & $30.91+2.05^{a}$ & $38.60 \pm 0.91^{b}$ & $41.58 \pm 2.27^{b}$ & 0.0015 \\
\hline TBARS, mg MDA/ kg & $0.95 \pm 0.10^{a}$ & $0.85 \pm 0.06^{a}$ & $0.87 \pm 0.13^{a}$ & 0.7078 \\
\hline
\end{tabular}

a,b Medias con diferente letra entre columnas, difieren entre sí ( $P \leq 0.05)$

La concentración de materia seca, proteína, grasa, cenizas de los cortes de carne evaluados se encuentran dentro de los rangos reportados previamente para bovinos Bos taurus, Bos indicus y cruces Bos indicus $x$ Bos taurus (Williams, 2007; Freitas et al., 2014; Teixeira et al., 2017). Los valores de TBARS se encontraron dentro de los rangos reportados para cortes de carne del músculo del lomo de bovinos Bos taurus entre el primer y tercer día de almacenamiento según lo descrito por Min et al. (2008), pero fueron mucho mayores que los encontrados $(0.5 \mathrm{mg} / \mathrm{kg}$ ) en cortes del musculo Longissimus lumborum de bovinos Bos taurus madurados por 14 días (Hyun-Wook et al ., 2016).

En cuanto al grupo racial, al igual que lo encontrado en este trabajo, Climaco et al., 2011 en Brasil, no se encontraron diferencias en la humedad, proteína, grasa y cenizas del músculo Longissimus dorsi de novillos puros Bos indicus Tabapuã y Bos taurus Bonsmara y cruces de novillos $1 / 2$ Bonsmara $+1 / 2$ Nelore y $1 / 2$ Bonsmara $+1 / 4$ Angus Rojo $+1 / 4$ N elore de 22 meses y $394 \mathrm{~kg}$ de peso vivo alimentados con ensilaje de caña de azúcar y un suplemento proteico energético y mineral comercial. Pero la concentración de proteína (23.5 a $24 \%$ ) y cenizas (3.7 a $4.0 \%$ ) del músculo del lomo de los diferentes grupos raciales evaluados fue mayor a la del presente estudio, aunque la de grasa fue mucho menor (0.5 a $1.0 \%$ ).

En forma similar a lo reportado en esta investigación, en Brasil, Moreira et al. (2003), en novillos Nelore (Bos indicus) y cruces de Nelore con Limousin y Girolando (Bos indicus $\times$ Bos taurus) finalizados en pastoreo con suplementación mineral, no encontraron efecto del grupo racial sobre la concentración de proteína cruda y cenizas, pero si en la de grasa, la cual fue mayor en novillos Nelore. Así mismo, Bressan et al. (2011) en Brasil en toros Bos taurus y Bos indicus comerciales con edades entre 26 y 40 meses finalizados en pastoreo de Brachiaria spp. y suplementación no encontraron diferencias en humedad y proteína del músculo Longissimus thoracis, pero al contrario encontraron mayor concentración de cenizas ( $0.1 \%$ más) y menor de grasa ( $0.7 \%$ menos) en toros B os taurus.

En Brasil Texeira et al. (2017) no encontraron diferencias en la humedad, proteína y cenizas, pero si en la grasa de los cortes del músculo Longissimus thoracis de toretes Nelore y Angus finalizados con suplementación de grano de maíz. Fernandes et al. (2009) similar a este estudio, cuando evaluaron la composición nutricional del músculo Longissimus lumborum de toros N elore y Canchim alimentados con dietas con base en caña de azúcar y dos niveles de concentrado en Brasil, no encontraron diferencias entre grupos raciales en la concentración de proténa y grasa; pero, al contrario, si observaron mayor concentración de cenizas y una tendencia a mayor humedad en el Canchim frente al el Nelore.

En Colombia Vásquez et al. (2007) a diferencia del presente trabajo, encontraron mayor concentración de grasa total en bovinos Brangus y Cebú Brahman comparados con bovinos criollos Romosinuano y cruces de Romosinauno $\times$ Cebú. Así mismo, Uzcátegui-Bracho et al. (2008) en filetes del músculo Longissimus madurados por 14 días de novillos criollo limonero de 36 meses de edad, que pastoreaban praderas de Echinochloa polystachia con suplementación, encontraron mayor concentración de proteína cruda, cenizas y menor de lípidos, en los filetes evaluados. 
Con relación a la concentración de colesterol total Huerta-Leidenz et al. (1996) en un estudio observacional en cortes del músculo Longissimus de bovinos Cebú y bovinos lecheros en pastoreo en Venezuela encontraron resultados similares a los de esta investigación. Pero otro trabajo realizado en Venezuela en novillos criollo Limonero de 36 meses, que se mantuvieron en pastoreo Echinochloa polystachia con suplementación, reportaron mayor concentración de colesterol $(58.13 \mathrm{mg} / 100 \mathrm{~g})$ en filetes del músculo Longissimus dorsi (Uzcátegui-Bracho et al., 2008).

La concentración de colesterol total en la carne puede depender del sistema de alimentación durante la ceba y su interacción con el grupo racial. En Brasil Bressan et al. (2011), en toros Bos taurus y Bos indicus comerciales observaron que las diferencias en colesterol total entre grupos raciales dependían del sistema de ceba. En sistemas de ceba en pastoreo, los resultados fueron similares a los de la presente investigación, debido a que la concentración de colesterol total del músculo Longissimus thoracis de toros Bos taurus fue mayor ( $45.5 \mathrm{mg} / 100 \mathrm{~g}$ ) que la de los Bos indicus (36.9 mg/ $100 \mathrm{~g}$ ), pero en bovinos finalizados con granos, el colesterol total de los toros Bos indicus presentó casi dos veces mayor concentración (65.8 mg/ 100 g) en comparación con los de bovinos B os taurus ( $38.8 \mathrm{mg} / 100 \mathrm{~g}$ ).

Lo anterior lo confirma un estudio realizado en Brasil (Rossato et al., 2009) donde se evaluó la concentración de colesterol total en el músculo Longissimus dorsi de bovinos Bos taurus y Bos indicus entre 30 a 36 meses de edad con pesos de canal entre 240 y $270 \mathrm{~kg}$ finalizados en lotes de engorde, encontrando mayor concentración en el Bos indicus ( $66.95 \mathrm{mg} / 100 \mathrm{~g}$ ), que en el Bos taurus (37.37 mg/ 100 g). Los valores reportados para el Cebú fueron el doble a los de la presente investigación muy posiblemente asociado a la dieta de finalización alta en energía.
Evaluaciones del efecto de la heterosis en toretes en Brasil (Gama et al., 2013) encontraron que en la ceba en pastoreo hay poco cambio en la concentración de grasa y colesterol total entre grupos raciales, pero en sistemas de ceba con el uso de granos la concentración de grasa y colesterol total disminuyen en los bovinos cruzados como lo reportaron Rosatto et al., 2009 y Bressan et al., 2011.

En contraste con la presente investigación, Moreira et al. (2003), no encontraron diferencias en el colesterol total entre cortes de carne de novillos Bos indicus (35.16 $\mathrm{mg} / 100 \mathrm{~g}$ ) y Bos taurus $\times$ Bos indicus $(39.64 \mathrm{mg} / 100 \mathrm{~g}$ ) cebados en pastoreo. Así mismo, trabajos realizados en Brasil (Bragagnolo y. Rodriguez-Amaya, 2003) en cortes del músculo Longissimus dorsi de bovinos Nelore (Bos indicus) y cruces de Canchim (3/ 8 Nelore $\times 5 / 8$ Charolais) no encontraron diferencias en la concentración de colesterol entre grupos raciales, aunque los valores fueron levemente superiores a los de la presente investigación con $40 \mathrm{mg} / 100 \mathrm{~g}$ para el Nelore y 43 mg/ $100 \mathrm{~g}$ para el Canchim, En un estudio de Brasil (Freitas et al., 2014) realizado en cortes del músculo Longissimus dorsi de novillos Hereford, $1 / 4$ Braford y $3 / 8$ Braford finalizados en pastoreo y lotes de engorde, tampoco reportaron diferencias entre grupos raciales, pero si mayor concentración de colesterol (47.17 a $48.66 \mathrm{mg} / 100 \mathrm{~g}$ ) que las observadas en la presente investigación (30.91 a 41.58 mg/ 100 g).

\section{Minerales en Carne}

En el Cuadro 3 se presenta la concentración de minerales de filetes de carne del músculo Longissimus dorsi. Los filetes de lomo de bovinos Cebú presentaron menor concentración de potasio $(p=0.0257)$ y zinc ( $p$ $=0.0079$ ) en comparación con el BON $\times$ C y SM $\times C$; el fósforo fue menor $(p=0.0335)$ y el magnesio tendió a ser menor $(p=0.0943)$ en el Cebú con respecto al $\mathrm{SM} \times \mathrm{C}$, pero no con el BON $\times$ C (Cuadro 3). Para el calcio, hierro y sodio no se encontraron diferencias entre grupos raciales ( $p>0.05$; Cuadro 3 ).

Cuadro 3. Concentración de Minerales en Cortes del Músculo Longissimus dorsi de Diferentes Grupos Raciales Bovinos del Piedemonte del Meta

\begin{tabular}{|c|c|c|c|c|}
\hline \multirow[t]{2}{*}{ Variable } & \multicolumn{4}{|c|}{ Grupo Racial } \\
\hline & Cebú & $\mathrm{BON} \times \mathrm{C}$ & $\mathrm{SM} \times \mathrm{C}$ & $\operatorname{Pr}>F$ \\
\hline Potasio, g/ 100g & $0.17 \pm 0.01^{b^{*}}$ & $0.22 \pm 0.02^{a}$ & $0.26 \pm 0.03^{a}$ & 0.0257 \\
\hline Fósforo, g/ 100g & $0.12 \pm 0.01^{\mathrm{b}}$ & $0.14 \pm 0.01^{a b}$ & $0.17 \pm 0.01^{a}$ & 0.0335 \\
\hline Sodio, g/ 100g & $0.05 \pm 0.00^{\mathrm{a}}$ & $0.04 \pm 0.00^{a}$ & $0.05 \pm 0.01^{a}$ & 0.4303 \\
\hline Calcio, mg/ 100g & $17.63 \pm 0.86^{a}$ & $20.14+1.80^{a}$ & $18.77 \pm 0.96^{a}$ & 0.4454 \\
\hline Magnesio, mg/ 100g & $18.21+1.30^{b}$ & $19.84 \pm 0.91^{\mathrm{ab}}$ & $22.97+2.06^{a}$ & 0.0943 \\
\hline Hierro, mg/ 100g & $5.62 \pm 0.32^{\mathrm{a}}$ & $7.24 \pm 0.57^{a}$ & $6.93 \pm 2.68^{a}$ & 0.4800 \\
\hline Zinc, mg/ 100g & $2.25 \pm 0.26^{b}$ & $3.21 \pm 0.31^{a}$ & $4.14 \pm 0.53^{a}$ & 0.0079 \\
\hline
\end{tabular}

*Medias con diferente letra entre columnas, difieren entre sí ( $\mathrm{P} \leq 0.05)$ 
La carne bovina es una de las fuentes más ricas de minerales para la alimentación humana, especialmente de hierro, zinc y selenio contribuyendo hasta en tres cuartos de las necesidades diarias por cada $100 \mathrm{~g}$ de carne consumida por día. Duckett et al. (2009), mencionan que las diferencias en la concentración mineral de la carne se deben en parte a la composición racial, los forrajes consumidos y prácticas de manejo que afectan la composición de la carne de los bovinos en pastoreo, pero en forma directa, la composición de minerales en la carne se asocia con el consumo de minerales y en bovinos en pastoreo con la concentración en los forrajes y el suelo (Leheska, 2008; Cabrera y Saadoun 2014).

En Venezuela Huerta-Leidenz et al. (2003) evaluaron en cortes del músculo Longissimus dorsi de toros, novillos y novillas de diferentes grupos raciales y sistemas de producción entre ellos bovinos lecheros con predominio de Holstein, Pardo Suizo (B os taurus), Doble Propósito (Bos indicus $\times$ Bos taurus), y mestizos de Cebú (Bos indicus), la concentración mineral que en promedio fueron similares a las de esta investigación para magnesio, $(21.62 \mathrm{mg} / 100 \mathrm{~g})$, potasio $(0.24 \mathrm{~g} / 100$ $\mathrm{g})$, y zinc $(4.13 \mathrm{mg} / 100 \mathrm{~g})$, mayores para fósforo (0.21 $\mathrm{g} / 100 \mathrm{~g})$, sodio, $(0.76 \mathrm{~g} / 100 \mathrm{~g})$ y menores para calcio $(2.77 \mathrm{mg} / 100 \mathrm{~g})$ y hierro $(1.93 \mathrm{mg} / 100 \mathrm{~g})$. En otro trabajo realizado en Venezuela (Uzcátegui-Bracho et al., 2008) en novillos criollo Limonero de 36 meses, que se mantuvieron en pastoreo Echinochloa polystachia con suplementación, se encontraron concentraciones similares de fósforo $(0.177 \mathrm{~g} / 100 \mathrm{~g})$, magnesio (18.56 $\mathrm{mg} / 100 \mathrm{~g})$ y zinc $(3.02 \mathrm{mg} / 100 \mathrm{~g})$, menores de hierro $(1.01 \mathrm{mg} / 100 \mathrm{~g})$, calcio $(2.2 \mathrm{mg} / 100 \mathrm{~g})$ y sodio (3.45 $\mathrm{mg} / 100 \mathrm{~g}$ ) y mayores de potasio $(325.1 \mathrm{mg} / 100 \mathrm{~g})$.

Así mismo, en investigaciones realizadas en Australia (Williams, 2007) y Brasil (Freitas, et al., 2014), la concentración de magnesio, zinc y sodio en cortes de músculo Longissimus dorsi de bovinos Bos taurus y Bos indicus $\times$ Bos taurus encontrada en el presente trabajo, fue similar, pero la de hierro mayor y la de fósforo menor. Para el potasio el estudio de Williams (2007) indica que la concentración fue menor pero el de Freitas et al. (2014) reporta mayor valor.

Llama la atención la concentración de hierro (5.62 a $7.24 \mathrm{mg} / 100 \mathrm{~g}$ ) encontrada en la presente investigación que dobla a la reportada previamente para la carne de bovinos de diferentes países suramericanos descrita en la revisión de Saadoun y Cabrera, (2013) para bovinos criollos (2.2 a $2.3 \mathrm{mg} / 100$ g) y cruces de criollo $\times$ Cebú $(2.0$ a $2.8 \mathrm{mg} / 100 \mathrm{~g}$ ) en Argentina, Holstein en Chile (1.0 a $2.0 \mathrm{mg} / 100 \mathrm{~g}$ ) y Hereford (1.8 a $4.6 \mathrm{mg} / 100 \mathrm{~g}$ ) y Braford (1.7 a 4.3 $\mathrm{mg} / 100 \mathrm{~g}$ ) en Uruguay.
Con relación al zinc en Uruguay, Cabrera et al. (2010) en siete diferentes cortes de carne incluyendo el músculo Longissimus dorsi de novillos Braford (3/ 8 Bos indicus $\times 5 / 8$ Bos taurus) y Hereford de 26 a 30 meses con $450 \mathrm{~kg}$ que se mantuvieron en pasturas naturales $y$ se finalizaron en praderas mejoradas, reportaron concentraciones de zinc para el Hereford ( 2.3 y 7.3 $\mathrm{mg} / 100 \mathrm{~g}$ ) y Braford (2.3 y $6.4 \mathrm{mg} / 100 \mathrm{~g}$ ) similares a los del presente trabajo.

Sin embargo, en este estudio la concentración de zinc encontrada en el Cebú ( $2.25 \mathrm{mg} / 100 \mathrm{~g}$ ) es inferior a la de los bovinos criollos cruzados. Este hallazgo puede reflejar la baja concentración de zinc en los suelos y praderas como lo mencionaron Cabrera et al. (2010) para bovinos Hereford y Braford en Uruguay. En la región de la Orinoquia donde se desarrolló el presente trabajo se encuentran suelos y pastos con baja concentración zinc (Flórez, 2012) y es posible que la cantidad del mineral disponible para absorción en el tracto gastrointestinal sea mayor en los bovinos criollos cruzados que en el Cebú, situación que también se pudo presentar para otros minerales como el potasio y el fósforo; sin embargo, los estudios de Ramos et al. (2012), no encontraron diferencias en la bio-accesibilidad del zinc en cortes de carne de bovinos Hereford y Braford.

El efecto del grupo racial en la concentración de minerales en la carne parece depender del tipo de alimentación durante la ceba. En un trabajo realizado en Brasil (Freitas et al., 2014) donde se evaluó el perfil mineral de músculo Longissimus dorsi de novillos Hereford, $1 / 4$ Braford (1/ 4 Nelore $\times 3 / 4$ Hereford), y $3 / 8$ Braford (3/ 8 Nelore $\times 5 / 8$ Hereford) finalizados en lotes de engorde y en pastoreo de praderas de Lolium multiflorum, Trifolium repens y Lotus corniculatus, se observó que la concentración de hierro fue mayor en la carne de bovinos $1 / 4$ Braford que en Hereford y 3/ 8 Braford. También se observó una interacción entre el grupo racial y el tipo de finalización, con concentraciones de zinc mayores en Hereford y $1 / 4$ Braford, en comparación con novillos que tenían mayor proporción de sangre Cebuína (3/ 8 Braford) finalizados en pastoreo. Contrario a lo observado en el estudio de Freitas et al. (2014), en la presente investigación no se observaron diferencias en la concentración de hierro por grupos raciales, pero si para magnesio y potasio (Cuadro 3). Para el sodio en forma similar a este estudio no se observaron diferencias entre grupos raciales.

Las menores concentraciones de fósforo, potasio y zinc y la tendencia de menor concentración en magnesio en los cortes de lomo del Cebú con relación al $\mathrm{SM} \times \mathrm{C}$ pueden ser además, el resultado de

ISSN-L 1022-1301. Archivos Latinoamericanos de Producción A nimal. 2021.29 (3-4): 163-179 
diferencias entre la concentración de grasa de la carne de estos grupos raciales (6.91 vs. $4.43 \%$; Cuadro 2), tal como se ha mencionado en otros trabajos (Leheska et al., 2008) y como lo sugiere Freitas et al. (2014) en cortes del músculo Longissimus dorsi de novillos Hereford, $1 / 4$ Bradford y 3/ 8 Bradford finalizados en pastoreo y lotes de engorde quienes describen correlaciones negativas entre el hierro, zinc, fósforo, sodio y potasio y la concentración de grasa. Bressan et al., 2011, en cortes del músculo Longissimus thoracis de toros Bos indicus y Bos taurus encontraron una correlación negativa $(r=-0,47)$ entre la concentración de grasa y las cenizas, aunque en ese estudio no se evaluaron las concentraciones de cada mineral.

\section{Conclusiones}

La evaluación de la calidad y la composición de los cortes de carne del músculo Longissimus dorsi de bovinos Cebú y los cruces de Sanmartinero $\times$ Cebú y Blanco Orejinegro $\times$ Cebú $(B O N \times C)$ en las condiciones de alimentación y manejo del Piedemonte del Meta, determinó que los filetes de bovinos $B O N \times C$ presentaron menor color y mayor decoloración. La evaluación sensorial de sabor realizada por panelistas entrenados encontró menor terneza y jugosidad en el $B O N \times C$. En cuanto a la composición nutricional se encontró que los cortes de carne del Cebú presentaron menor concentración de colesterol total, potasio y zinc.
Los resultados presentados muestran diferencias en la calidad sensorial y la composición nutricional de la carne asociadas al grupo racial y al manejo durante el levante y la ceba. Se deben realizar investigaciones posteriores que involucren la calidad organoléptica y composicional de diferentes cortes de la canal bovina en bovinos criollos y sus cruces con esquemas de manejo del pastoreo, suplementación estratégica y con fuentes alternativas de alimentación que permitan posicionar la carne criolla y sus cruces en el mercado colombiano.
Los autores expresan sus más sinceros agradecimientos al personal de la planta de Alimentar Beef Market en Bogotá que apoyaron el faenado de los bovinos, y el suministro de información del desposte, al personal del frigorífico Guadalupe en Bogotá por el apoyo con el faenado y desposte de bovinos. Los autores también agradecen al Ministerio de Agricultura y Desarrollo Rural (MADR) de Colombia y la Corporación Colombiana de Investigación Agropecuaria, AGROSA VIA, por la financiación de la meta de investigación que generó la información para

\section{Literatura Citada}

A berle, E. D., E. S. Reeves, M. D. Judge, R. E. Hunsley, and T. W. Perry. 1981. Palatability and muscle characteristics of cattle with controlled weight gain: Time on a high energy diet. J. A nim. Sci. 52:757-763. https:/ / doi.org/ 10.2527/ jas1981.524757x

American Meat Science Association (AMSA). 2016. Research guidelines for cookery, sensory evaluation, and instrumental tenderness measurements of meat. 2nd ed. American Meat Science Association, Champaign, IL. $106 \quad$ pp. https:/ / meatscience.org/ docs/ defaultsource/ publications-resources/ amsa-sensory-andtenderness-evaluation-guidelines/ researchguide/ amsa-research-guidelines-for-cookery-andevaluation-1-02.pdf?sfvrsn=4c6b8eb3_2

A merican meat Science Association. AMSA. 2012. Meat American meat Science Association. AMSA. 2012. Meat Color Measurement Guidelines. 2012. American Meat Science Association Champaign, Illinois USA. $136 \mathrm{pp}$. https:/ / meatscience.org/ docs/ defaultsource/ publications-resources/ hottopics/ download-the-ebook-format-pdf-of-themeat-color-measurement-

AOAC. 1990. Official Methods of Analysis of the Association of Official Analytical Chemists. 15th edition Arlington, VA, USA.

AOAC. 1997. Official Methods of Analysis of the Association of Official Analytical Chemists. 16th edition A rlington, VA, USA.

Astruc, T. 2014. Connective tissue: structure, function, and influence on meat quality. Encyclopedia of Meat Sciences, 1 (2ème ed.), Elsevier Ltd, 2014. ffhal02801237f. https:/ / hal.inrae.fr/ hal02801237/ document consultado 25 de noviembre de 2020.

Baublits, R. T., Pohlman, F. W., Brown, A. H Jr., Yancey, E. J., Johnson, Z. B. 2006a. Impact of muscle type and sodium chloride concentration on the quality, sensory, and instrumental color 
characteristics of solution enhanced whole-muscle $\begin{array}{lll}\text { beef. } \quad \text { Meat } & \text { 52(4):704-12. }\end{array}$ https:/ / doi.org/ 10.1016/ j.meatsci.2005.09.023

Baublits, R., Pohlman, F., Brown Jr., A., Johnson, Z. 2006b. Enhancement with varying phosphate types, concentrations, and pump rates, without sodium chloride on beef biceps femoris quality and sensory characteristics. Meat Science. 72(3), 404-414. https:/ / doi.org/ 10.1016/ j.meatsci.2005.08.006

Boleman, S. J., Boleman, S. L., Miller, R. K., Taylor, J. F., Cross, H. R., Wheeler, T. L., Koohmaraie, M., Shackelford, S. D., Miller, M. F., West, R. L., Johnson, D. D., and Savell, J. W. 1997. Consumer evaluation of beef of known categories of tenderness. J. Anim. Sci. 75:1521-1524. https:/ / doi.org/ 10.2527/ 1997.7561521x

Bragagnolo, N., Rodriguez-Amaya, D. 2003. New data on the total lipid, cholesterol and fatty acid composition of raw and grilled beef Longissimus dorsi Archivos Latinoamericanos de N utrición 53(3): 312-319. ISSN 0004-0622.

Bressan, M. C., Rodrigues, E. C., Rossato, L. V., Ramos, E. M., da Gama, L. T. 2011. Physicochemical properties of meat from Bos taurus and Bos indicus. R. Bras. Zootec., 40(6): 1250 - 1259.

Bruce, H. L., Roy, B. C. 2019. Meat science and muscle biology symposium: biological influencers of meat palatability: Production factors affecting the contribution of collagen to beef toughness 1,2 . J. $\begin{array}{lll}\text { Anim } & \text { Sci. } & \text { 97(5): }\end{array}$ https:/ / doi.org/ 10.1093/ jas/ skz097

Bueso, M. E. Garmyn, A. J., O’Quinn, T. G., Brooks, J. C., Brashears, M. M., and Miller, M. F. 2018. Comparing Honduran and United States Consumers' Sensory Perceptions of Honduran and U.S. Beef Loin Steaks. Meat and Muscle Biology 2(1): 233 241. https:/ / doi.org/ 10.22175/ mmb2018.03.0003

Cabrera, M. C. and A. Saadoun. 2014. An overview of the nutritional value of beef and lamb meat from South A merica. Meat Sci. 98, 435 - 444.

Cabrera, M.C., Ramos, A., Saadoun, A., Brito, G. 2010. Selenium, copper, zinc, iron and manganese content of seven meat cuts from Hereford and Braford steers fed pasture in Uruguay. Meat Sci. 84(3): 518 - 528. https:/ / doi.org/ 10.1016/ j.meatsci.2009.10.007

Cafe L. M., Robinson, D. L., Ferguson, D. M., Mclntyre, B. L., Geesink, G.H., Greenwood P. L. 2011. Cattle temperament: Persistence of assessments and associations with productivity, efficiency, carcass and meat quality traits. J A nim. Sci. 89:1452 - 1465.

Chriki, S., Renand, G., Picard, B., Micol, D., Journaux, L., Hocquette, J. F. 2013. Meta-analysis of the relationships between beef tenderness and muscle characteristics. Livestock Science. 155(2-3): 424-434. https:/ / doi.org/ 10.1016/ j.livsci.2013.04.009.

Climaco, S. M., Ribeiro, E. L. A., Mizubuti, I. Y., da Silva, L. D. F., de Freitas Barbosa, M. A. A., de Oliveira Ramos, B. M., Constantino, C. 2011. Características de carcaça e qualidade da carne de bovinos de corte de quatro grupos genéticos terminados em confinamento. R. Bras. Zootec., 40 (12): 2791 - 2798.

Connell, J., Huerta-Leidenz, N., Rodas-González, A. 2002. Rendimiento del cuarto trasero y calidad sensorial del músculo longissimus de novillos zulianos. Revista Científica FCV-LUZ, 12Suplemento $\quad 2: \quad 622-625$. http:/ / www.saber.ula.ve/ handle/ 123456789/ 28202

Crouse, J. D.; Cundiff, L. V.; Koch R, M.; Koohmaraire, M., and Seideman, S. C. 1989. Comparison of Bos indicus and Bos taurus inheritance for carcass beef characteristics and mean palatability. J. Anim. Sci. 67:2661-2668.

Cuvelier, C., Clinquart, A., Hocquette, J.F., Cabaraux, J.F., Dufrasne, I., Istasse, L., Hornick, J.L. 2006. Comparison of composition and quality traits of meat from young finishing bulls from belgian blue, limousin and aberdeen angus breeds. Meat Sci., 74, 522-531.

Duckett, S. K., Neel, J. P. S., Fontenot, J. P., Clapham, W. M. 2009. Effects of winter stocker growth rate and finishing system on: III. Tissue proximate, fatty acid, vitamin, and cholesterol content, J. A nimal Sci. 87(9): 2961-2970, https:/ / doi.org/ 10.2527/ jas.20091850

Emerson, M. R., D. R. Woerner, K. E. Belk, and J. D. Tatum. 2013. Effectiveness of USDA instrumentbased marbling measurements for categorizing beef carcasses according to differences in longissimus muscle sensory attributes. J. Anim. Sci. 91:10241034. https:/ / doi.org/ 10.2527/ jas.2012-5514

Fernandes, A. R. M., Sampaio, A. A. M., Henrique, W., de Oliveira, E. A., Oliveira, R. V., Leonel, F. R. 2009. Composição em ácidos graxos e qualidade da carne de tourinhos Nelore e Canchim alimentados com dietas à base de cana-de-açúcar e dois níveis de concentrado. R. Bras. Zootec., 38(2): 328-337.

Flórez D. H. 2011. Estrategias para mejorar la calidad de la carne bovina en Colombia. Memorias Primer congreso internacional de ciencia y tecnología de la carne. Bogotá 15-18 de marzo de 2011. 34pp.

Flórez D. H. 2012. Requerimientos y recomendaciones de suplementación con minerales en bovinos. Capítulo 4. En: Manejo de la nutrición mineral en sistemas ganaderos de los Llanos Orientales de Colombia. Corpoica, Ministerio de Agricultura y Desarrollo Rural. Eds. Álvaro Rincón Castillo; César 
Augusto Jaramillo Salazar. Pp. 81-110. ISBN: 978958-740-115-8.

Flórez Díaz, H., León Llanos M., Moreno, E., Ballesteros C. H., Martínez Correal, G. 2015b. Concurso de Ganado Gordo Feria Malocas 2015. Revista el Cebú, Mayo Junio. 404: 50 - 56. ISSN : 0122 - 2201

Flórez Díaz, H.; León Llanos, L.M; Ballesteros Chavarro, H.; Castañeda, S.; Moreno Moreno, E.; Martínez Correal, G.; Torres Cardona, J.C.; Peña Castellanos, F. 2015a. Calidad de la carne de bovinos criollos y europeos y sus cruces con Cebú en las condiciones de la Orinoquia colombiana. Revista AICA, 4:12-15. ISSN (versión impresa): 2253-7325; ISSN (versión web online): 2253-9727

Flórez Díaz, H.; Martínez Correal, G.; Ballesteros Chavarro, H.; León Llanos, L.M.; Castañeda, S.; Moreno Moreno, E.; Arias Castellanos, L.E.; Torres Cardona, J.C.; Rodríguez Rojas, C.A.; Peña Castellanos, F.; \& Uribe Botero, A. 2014. Rendimiento en carne de bovinos criollos y europeos y sus cruces con Cebú en las condiciones de la Orinoquia colombiana. Revista AICA, 4:12-15. ISSN (versión impresa): 2253-7325; ISSN (versión web online): 2253-9727.

Font-i-Furnols, M., and Guerrero, L. 2014. Consumer preference, behavior and perception about meat and meat products: An overview. Meat Science, 98, 361-371. meatsci.2014.06.025

Francisco, C. L., F. D. Resende, J. M. B. Benatti, A. M. Castilhos, R. F. Cooke, and A. M. Jorge. 2015. Impacts of temperament on Nellore cattle: Physiological responses, feedlot performance, and carcass characteristics. J. Anim. Sci. 93:5419-5429. https:/ / doi.org/ 10.2527/ jas.2015-9411

Freitas A.K., Lobato, J. F. P., Cardoso, L. L., Tarouco, J. J. U., Vieira, R. M., Dillenburg, D. R., Castro, I. 2014. Nutritional composition of the meat of Hereford and Braford steers finished on pastures or in a feedlot in southern Brazil. M eat Science. 96:353-360.

Fruet, A. P. B., Trombettaa, F., Stefanelloa, F. S., Speronia, C. S., Donadela, J. Z., De Souzab, A. N. M., Rosado Júniorb, A., Tonettob, C. J., Wagnera, R., De Melloc, A., Nörnberga, J. L. 2018. Efects of feeding legume-grass pasture and diferent concentrate levels on fatty acid profile, volatile compounds, and of-flavor of the M. Iongissimus thoracis. Meat Science. 140: 112-118.

Gama, L. T., Bressan, M.C., Rodrigues, E.C., Rossato, L.V., Moreira, O.C., Alves, S.P, Bessa, R.J.B. 2013. Heterosis for meat quality and fatty acid profiles in crosses among Bos indicus and Bos taurus finished on pasture or grain. Meat Science 93: 98-104.
Girolami, A., F. Napolitano, D. Faraone, and A. Braghieri. 2013. Measurement of meat color using a computer vision system. Meat Sci. 93:111-118. https:/ / doi.org/ 10.1016/ j.meatsci.2012.08.010

Gómez, J. F. M., Saran Netto, A., A ntonelo, D. S., Silva, J., Sene, G. A., Silva, H. B., Dias, N. P., Leme, P. R. and Silva, S. L. 2017. Effects of immunocastration on the performance and meat quality traits of feedlotfinished Bos indicus (Nellore) cattle. Animal Production Science. 59(1) 183-190.

Gracia A, de Magistris T, 2013. Preferences for lamb meat: A choice experiment for Spanish consumers. Meat Sci 396-402. http:/ / dx.doi.org/ 10.1016/ j.meatsci.2013.05.006

Hardcastle, N., Garmyn, A. J., Legako, J. F., Brashears, M. M. and Miller M. F. 2017. Honduran Consumer Perception of Palatability of Enhanced and NonEnhanced Beef from Various Finishing Diets. Meat and Muscle Biology 1(3):94

Hocquette, J.F., Renand, G., Levéziel, H., Picard, B., Cassar-Malek, I. 2005. Genetic effects on beef meat quality. The science of beef quality. School of Clinical Veterinary Science, University of Bristol. 1320. https:/ / www.agrireseau.net/ bovinsboucherie/ documents/ BQ_May05p.pdf Consultado 24 de julio 2020

Huerta-Leidenz, N, Ruíz-Ramírez, J. L., ArenasMoreno, L., Jerez-Timaure, N., Márquez, E., Muñoz, B. 1996. Cholesterol content in longissimus muscle beef from slaughter cattle in Venezuela. Arch Latinoam Nutr. 46(4):329-333.

Huerta-Leidenz, N., Arenas de Moreno, L. MoronFuenmayor, O., Uzcátegui-Bracho, S. 2003. Composición mineral del músculo longissimus crudo derivado de canales bovinas producidas y clasificadas en Venezuela. Archivos Latinoamericanos de Nutrición, 53(1): 96-101. Recuperado en 27 de julio de 2020.

Huerta-Leidenz, N., Rodas González, A., Smith, G. 2004. Effect of vacuum aging and influence of sire on palatability of beef longissimus from grass-fed F1 Senepol x Zebu bulls. Revista Científica, FCV-LUZ, 14(3): 263-269.

Hyun-Wook, K., Derico, S., Yun-Sang, Ch., and Yuan H. B. K. 2016. Rapid Discoloration of Aged Beef Muscles after Short-Term/ Extreme Temperature Abuse during Retail Display. Korean J. Food Sci. An. 36(3): 343-351.

King, D. A., C. E. Schuehle Pfeiffer, R.D. Randel, T.H. Welsh Jr, R.A. Oliphint, B.E. Baird, K.O. Curley Jr, R.C. Vann, D.S. Hale, and J.W. Savell. 2006. Influence of animal temperament and stress responsiveness on the carcass quality and beef tenderness of feedlot cattle. Meat Science 74: 546556. 
King, D. A., Shackelford, S. D., Wheeler, T. L. 2014. Animal variation in lean color stability. Proceedings 67th Annual Reciprocal Meat Conference. American Meat Science Association. $5 p$. https:/ / meatscience.org/ docs/ defaultsource/ publications-resources/ rmc/2014/07_king .pdf?s fvrsn=0 Consultado 23 de julio de 2020.

Koohmaraie, M., Geesink, G. H. 2006. Contribution of postmortem muscle biochemistry to delivery of consistent meat quality with particular focus on the calpain system. Meat Science. 74(1): 34 - 43. https:/ / doi.org/ 10.1016/ j.meatsci.2006.04.025 .

Leheska, J. M., Thompson, L. D., Howe, J. C., Hentges, E., Boyce, J., Brooks, J. C., Shriver, B., Hoover, L., Miller, M. F. 2008. Effects of conventional and grassfeeding systems on the nutrient composition of beef, Journal of Animal Science, 86 (12): 3575-3585, https:/ / doi.org/ 10.2527/ jas.2007-0565

León-Llanos, L. M., Flórez-Díaz, H. 2016. La importancia del temperamento en la producción de ganado de carne bovina. ORIN OQUIA : 20:2. 55 - 63. https:/ / doi.org/ 10.22579/ 20112629.354

Lippi, G., C. Mattiuzzi, and F. Sanchis-Gomar. 2015. Red meat consumption and ischemic heart disease. A systematic literature review. Meat Sci. 108:32-36. https:/ / doi.org/ 10.1016/ j.meatsci.2015.05.019

Lorenzen, C. L., T. R. Neely, R. K. Miller, J. D. Tatum, J. W. Wise, J. F. Taylor, M. J. Buyck, J. O. Reagan, and J. W. Savell. 1999. Beef customer satisfaction: Cooking method and degree of doneness effects on the top loin steak. J. Anim. Sci. 77:637-644. https:/ / doi.org/ 10.2527/ 1999.773637x

Lucherk, L. W., T. G. O'Quinn, J. F. Legako, R. J. Rathmann, J. C. Brooks, and M. F. Miller. 2016. Consumer and trained panel evaluation of beef strip steaks of varying marbling and enhancement levels cooked to three degrees of doneness. Meat Sci. 122:145-154.

https:/ / doi.org/ 10.1016/ j.meatsci.2016.08.005

Malheiros, J. M., de Almeida Lorenço, de Oliveira, J. P.; H. N; Galvão de Albuquerque L., Loyola Chardulo, L. A. 2015. Meat quality and carcass traits measured in Longissimus thoracis muscle of the feedlot animals. A bstract. Archivos Latinoamericanos de Producción A nimal. 23. Suplemento 1. p13.

Mancini, R.A., and M. Hunt. 2005. Current research in in meat color. Meat Sci. 71:100-121. https:/ / doi.org/ 10.1016/ j.meatsci.2005.03.003

Martínez C. G. y F. Gómez G. 2006. Calidad de canales en novillos Cebú y sus cruces con BON y Charoláis. Revista ACOVEZ Vol. 36 No. 2. / Edición 102 / Julio de 2006. p. 8-13.

Martins, T. S., Sanglard, L. M., Silva, W., Chizzotti, M. L., Rennó, L. N., Serão, N. V., Silva, F. F., Guimarães, S. E., Ladeira, M. M., Dodson, M. V. et al. 2015.
Molecular factors underlying the deposition of intramuscular fat and collagen in skeletal muscle of Nellore and Angus cattle. Plos One. 10: e0139943. https:/ / doi.org/ 10.1371/ journal. pone.0139943

May, M.L., Dikeman, M.E., Schalles, R. 1977. Longissimus muscle histological characteristics of Simmental $\times$ Angus, Hereford $\times$ Angus and Limousin $\times$ Angus crossbred steers as related to carcass composition and meat palatability traits. J. Anim. Sci., 44, 571-580

Merle, S., Sencleer, J., Rodas-González, A., González, J., Mansutti, D. Huerta-Leidenz, N. 2004. Comparación de machos enteros búfalos de agua (Bubalus bubalis) vs vacunos acebuados en características al sacrificio, de la canal, rendimiento carnicero y palatabilidad del longissimus. Arch. Latinoam. Prod. Anim. 12(3): 112-120.

https:/ / ojs.alpa.uy/ index.php/ ojs_files/ article/ vie w/ 21

Mestre Prates, J. A.; Gonçalves Quaresma, M. A.; Branquinho Bessa, R. J.; Andrade Fontes, C. M.G Cristina M.P. Mateus Alfaia. 2006. Simultaneous HPLC quantification of total cholesterol, tocopherols and $\beta$-carotene in Barrosã-PDO veal Food Chemistry, Volume 94, Issue 3, February 2006, Pages 469-477.

Miguel, G. Z., M. H. Faria, R. O. Roça, C. T. Santos, S. P. Suman, A. B. G. Faitarone, N. L. C. Delbem, L. V. C. Girao, J. M. Homem, E. K. Barbosa, L. S. Su, F. D. Resende, G. R. Siqueira, A. D. Moreira, and T. V. Savian. 2014. Immunocastration improves carcass traits and beef color attributes in Nellore and Nellore $\times$ Aberdeen Angus crossbred animals finished in feedlot. Meat Sci. 96:884-891. https:/ / doi.org/ 10.1016/ j.meatsci.2013.08.030

Miller, M. F., Carr, M. F., Ramsey, C. B., Crockett, K. L., and Hoover, L. C. 2001. Consumer thresholds for establishing the value of beef tenderness. J. Anim. Sci. 79:3062-3068.

Min, B. R., Nam, K. C., Cordray J. C., Ahn D. U. 2008.Factors Affecting Oxidative Stability of Pork, Beef, and Chicken Meat. Animal Science Research Reports. Digital Repository. Iowa State University. $5 p$.

Moreira, F.B., N.E. de-Souza, M. Matsushita, I.N. doPrado, and W.G. do-Nascimento. 2003. Evaluation of carcass characteristics and meat chemical composition of Bos indicus and Bos indicus $x$ Bos taurus crossbred steers finished in pasture systems. Braz. Arch. Biol. Technol. 46:609-616. https:/ / doi.org/ 10.1590/ S1516-89132003000400016

Neethling, N. E., S. P. Suman, G. O. Sigge, L. C. Hoffman, and M. C. Hunt. 2017. Exogenous and endogenous factors influencing color of fresh meat 
from ungulates. Meat and Muscle Biol. 1:253-275. https:/ / doi.org/ 10.22175/ mmb2017.06.0032

Nyquist, K. M., T. G. O'Quinn, L. N. Drey, L. W. Lucherk, J. C. Brooks, M. F. Miller, and J. F. Legako. 2018. Palatability of beef chuck, loin, and round muscles from three USDA quality grades. J. Anim. Sci.

https:/ / doi.org/ 10.1093/ jas/ sky305

Pavan, E., and Duckett, S. K. 2019. Longissimus Muscle Composition and Palatability of Grazing Steers Supplemented with Corn Oil or Corn Grain. Meat and Muscle Biology 3(1):181-193. https:/ / doi.org/ 10.22175/ mmb2018.10.0030

Phelps, K. J., Johnson, D. D., Elzo, M. A., Paulk, C. B, Gonzalez, J. M. 2017. Effect of Brahman genetics on myofibrillar protein degradation, collagen crosslinking, and tenderness of the longissimus lumborum. J Anim Sci. 95(12):5397-5406. https:/ / doi.org/ 10.2527/ jas2017.2022

Purslow, P. P. 2005. Intramuscular connective tissue and its role in meat quality. Meat Science. 70(3): 435447. ISSN

0309-1740.

https:/ / doi.org/ 10.1016/ j.meatsci.2004.06.028

Ramos, A., Cabrera, M. C. and Saadoun, A. 2012. Bioaccessibility of $\mathrm{Se}, \mathrm{Cu}, \mathrm{Zn}, \mathrm{Mn}$ and $\mathrm{Fe}$, and heme iron content in unaged and aged meat of Hereford and Braford steers fed pasture. Meat Sci. 91:116-124. https:/ / doi.org/ 10.1016/ j.meatsci.2012.01.001

Rodas-González, A., Vergara-López, J., Arenas de Moreno, L., Huerta-Leidenz, N., Leal M., Pirela, M. F. 2007. Efecto de la Suplementación y Maduración de Carnes al Vacío Sobre la Palatabilidad del Longissimus de N ovillos Criollo Limonero Cebadas a Pastoreo. Rev. Cient. (Maracaibo) 17(3): 280-287. http:/ / ve.scielo.org/ scielo.php?script=sci_arttext\& pid=S0798-22592007000300011\&lng=es.

Rossato, L. V., Bressan, M. C., Rodrigues, E. C., Carolino, M. I. Alves de Carvalho M., Bessa, R. J. B., Alves, S. P. P. 2009. Composição lipídica de carne bovina de grupos genéticos taurinos e zebuínos terminados em confinamento. Revista Brasileira de Zootecnia 38(9): 1841-1846.

Rossi, R., Pastorelli, G., Cannata, S., Tavaniello, S., Maiorano, G., Corino, C. 2013. Effect of long-term dietary supplementation with plant extract on carcass characteristics meat quality and oxidative stability in pork. Meat Science 95: 542-548. https:/ / doi.org/ 10.1016/ j.meatsci.2013.05.037

Roy, B. C., Sedgewick, G., Aalhus, J. L., Basarab, J. A. and Bruce, H. L. 2015. Modification of mature nonreducible collagen cross-link concentrations in bovine $\mathrm{m}$. Gluteus medius and semitendinosus with steer age at slaughter, breed cross and growth promotants. Meat Sci. 110:109- 117. https:/ / doi.org/ 10.1016/ j.meatsci.2015.07.008

Saadoun, A., Cabrera, M. C. 2013. Calidad nutricional de la carne bovina producida en Uruguay. Arch. Latinoam. Prod. Anim. 21 (2):119-130. https:/ / ojs.alpa.uy/ index.php/ ojs_files/article/ vie w/ 2251

Salim, A. P., Suman, S. P., Viana, F. M., Monteiro, M. L. G., Panzenhagen, P. H. N., Canto, A. C., and ConteJunior, C. A. 2019. Harvest Method Influences Color Stability of Longissimus Lumborum Steaks from Bos indicus Cattle. Meat and Muscle Biology 3(1):33-41. https:/ / doi.org/ 10.22175/ mmb2018.07.0021

SAS (Statistical analysis system institute). SAS STAT User's Guide. Version 9.2. Second edition. SAS Institute Inc. Cary. North Carolina, USA. 2009.

Scollan, N., J.F. Hocquette, K. Nuernberg, D. Dannenberger, I. Richardson, and A. Moloney. 2006. Innovations in beef production systems that enhance the nutritional and health value of beef lipids and their relationship with meat quality. Meat Sci.

74:17-33.

https:/ / doi.org/ 10.1016/ j.meatsci.2006.05.002

Seidemani, S. C., Cross, H. R., Smithl G. C. and Durlandj, P. R. 1984. Factors associated with fresh meat color: A review. Journal of Food Quality. 6: 211-237.

Suman, S. P., M. C. Hunt, M. N. Nair, and G. Rentfrow. Rentfrow. 2014. Improving beef color stability: Practical strategies and underlying mechanisms. Meat Sci. 98:490-504. https:/ / doi.org/ 10.1016/ j.meatsci.2014.06.032

Teixeira, P. D., Oliveira, D. M., Chizzottic, M. L., Chalfun-Juniord, A., Coelhoa, T. C., Gionbellia, M. P., Paivae, L. V., Carvalhoa, J. R. R., Ladeira, M. M. 2017. Subspecies and diet affect the expression of genes involved in lipid metabolism and chemical composition of muscle in beef cattle. Meat Science 133: $\quad 110 \quad-118$. https:/ / doi.org/ 10.1016/ j.meatsci.2017.06.009

Udomkun, P., Ilukor, J., Mockshell, J., Mujawamariya, G., Okafor, C. Bullock, R., Nanahungu, N., Vanlauwe, B. 2018. What are the key factors influencing consumers' preference and willingness to pay for meat products in Eastern DRC? Food Science \& Nutrition. 6: 2321-2336. https:/ / doi.org/ 10.1002/ fsn3.813

Uzcátegui-Bracho, S., Rodas-González, A., Hennig, K., Arenas de Moreno, L., Leal, M., Vergara-López, J. et al. 2008. Composición proximal, mineral y contenido de colesterol del músculo Longissimus dorsi de novillos criollo limonero suplementados a pastoreo. Rev. Cient. (Maracaibo) 18(5): 589-594. 
Van Elswyk, M. E., McNeill, S. H. 2014. Review. Impact of grass/ forage feeding versus grain finishing on beef nutrients and sensory quality: The U.S. experience. Meat Science 96(1): 535-540. https:/ / doi.org/ 10.1016/ j.meatsci.2013.08.010

Vásquez, R. E., H. Ballesteros, C. A. Muñoz. 2007. Factores asociados con la calidad de la carne. II parte: perfil de ácidos grasos de la carne bovina en 40 empresas ganaderas de la región Caribe y el Magdalena M edio. Revista Corpoica. 8:66-73.
Vestergaard, M., Oksbjerg, N., Henckel, P. 2000. Influence of feeding intensity, grazing and finishing feeding on muscle fibre characteristics and meat colour of semitendinosus, longissimus dorsi and supraspinatus muscles of young bulls. Meat Sci., 54, 177-185. 1740(99)00098-4 https:/ / doi.org/ 10.1016/ S0309-

Williams, P. G. 2007. Nutritional composition of red meat. Faculty of Science, Medicine and Health. University of Wollongong. Australia. Research Online.

http:/ / citeseerx.ist.psu.edu/ viewdoc/ download?d oi $=10.1 \cdot 1 \cdot 672.3304 \&$ rep $=$ rep $1 \&$ type $=p d f$ Consultada 29 octubre de 2016 . 